\title{
Working towards a 10-Year Aboriginal Health Plan: the Centre for Aboriginal Health initiatives
}

\author{
Geraldine Wilson $^{\mathrm{A}, \mathrm{C}}$ and Jessica Stewart ${ }^{\mathrm{B}}$ \\ ${ }^{\mathrm{A}}$ Centre for Aboriginal Health, NSW Ministry of Health \\ ${ }^{\mathrm{B}}$ Centre for Epidemiology and Evidence, NSW Ministry of Health \\ ${ }^{\mathrm{C} C o r r e s p o n d i n g ~ a u t h o r . E m a i l: ~ g w i l s @ d o h . h e a l t h . n s w . g o v . a u ~}$
}

The Centre for Aboriginal Health within the NSW Ministry of Health is responsible for policy development and strategic planning aimed at improving the health of Aboriginal people in NSW. The work of the Centre is guided by both national and NSW policies. In 2007, the Council of Australian Governments (COAG) agreed to a partnership between all levels of government with the aim of addressing Aboriginal and Torres Strait Islander health disadvantage through the National Indigenous Reform Agreement (NIRA). ${ }^{1}$ The Agreement includes objectives, outcomes, outputs, performance indicators and performance benchmarks agreed to by COAG, and links to other national agreements such as the $\$ 1.58$ billion National Partnership Agreement on Closing the Gap in Indigenous Health Outcomes. ${ }^{2}$ The NSW Government committed $\$ 180.38$ million over 4 years towards this agreement, to be implemented through the Centre for Aboriginal Health, including coordinating the delivery of a range of initiatives involving Local Health Districts, Aboriginal Community Controlled Health Services and the Aboriginal Health and Medical Research Council NSW (AH\&MRC).

In 2011 the NSW Government released NSW 2021, ${ }^{3}$ a state plan in which priority health issues and targets for achieving improvements to Aboriginal health are identified. Also in 2011, the NSW Government committed to the development of a 10-year Aboriginal health plan for NSW which the Centre for Aboriginal Health, in partnership with the AH\&MRC, is developing through statewide consultations.

To meet these policy commitments, the Centre for Aboriginal Health works in partnership with other NSW Ministry of Health branches to ensure statewide policies and programs meet the needs of Aboriginal people, including the Aboriginal Maternal and Infant Health Service, and the Chronic Care for Aboriginal People program (both of which are highlighted in this special edition) as well as programs that target tobacco, injury, workforce, housing and oral health. The Centre also develops specific Aboriginal health policies and programs needed to address gaps in the system that may not be the clear responsibility of other branches.

The following six short reports describe programs that address health promotion, family health, ear health, chronic disease, and improving data quality in both NSW hospitals and Aboriginal Community Controlled Health Services. These examples highlight different strategies used for engaging with the health system, including:

- Strategic policy development: The NSW Health Aboriginal Family Health Strategy ${ }^{4}$ and Aboriginal and Torres Strait Islander Origin - Recording of information of patients and clients (Policy Directive 2005-547)

- Specific program funding for priority areas: Health promotion program in Local Health Districts, the Chronic Care Service Enhancement Program in Aboriginal Community Controlled Health Services, and population health Ear Health program

- Research funding to develop evidence: Improving identification of Aboriginal patients and the cultural competency of the health workforce in NSW hospitals.

Identifying the suitability and effectiveness of these different strategies for achieving health gains in different areas will be important for the implementation of the 10-year Aboriginal health plan. Strategic policy and effective monitoring and accountability systems will be of increasing importance as responsibility for achieving Aboriginal health gains is further devolved to Local Health Districts. The coordination of statewide programs that specifically target equity issues across the health system for meeting the needs of Aboriginal people will also remain critical for developing evidence of best practice. The implementation of the same program in more than one Local Health District, Aboriginal Community Controlled Health Service or community provides opportunities for rigorous evaluation to determine the effectiveness of the program in improving health service delivery and health outcomes. The evidence produced can then be used to inform programs across NSW.

The Centre for Aboriginal Health continues to work to create opportunities to close the gap in health between Aboriginal and non-Aboriginal people by working in partnership with the Aboriginal Community Controlled Health Services and Local Health Districts. This collection of reports describes progress being made in addressing key Aboriginal health issues in NSW. 


\section{References}

1. Council of Australian Governments. National Indigenous Reform Agreement. Canberra: COAG; 2008. Available at: http://www.facs.gov.au/sa/indigenous/progserv/ctg/Pages/ NIRA.aspx (Cited 7 February 2012).

2. Council of Australian Governments. National Partnership Agreement on Closing the Gap in Indigenous Health Outcomes. 1 July 2009. Available at: http://www.coag.gov.au/ intergov_agreements/federal_financial_relations/docs/ national_partnership/NP_closing_the_Gap_indigenous_ health_outcomes.pdf (Cited 7 February 2012).
3. NSW Department of Premier and Cabinet. NSW 2021. A plan to make NSW number one. Sydney: NSW Government; 2011.

4. Health NSW. Aboriginal Family Health Strategy 2011-2016. Responding to family violence in Aboriginal communities. Sydney: Centre for Aboriginal Health; 2011. Available at: http:// www.health.nsw.gov.au/pubs/2011/aboriginal_family_health_. html (Cited 5 December 2011).

5. NSW Health. Aboriginal and Torres Strait Islander Origin Recording of information of patients and clients (PD 2005-547). Available at: http://www.health.nsw.gov.au/policies/pd/2005/ pdf/PD2005-547.pd (Cited 27 March 2012).

\section{NSW Aboriginal Health Promotion Program: lessons learned and ways forward}

\section{Ian Raymond ${ }^{\mathrm{A}}$, Catriona McDonnell ${ }^{\mathrm{A}}$ and Geraldine Wilson ${ }^{\mathrm{A}}$}

${ }^{\mathrm{A}}$ Centre for Aboriginal Health, NSW Ministry of Health

Chronic disease is one of the most significant health issues in Aboriginal communities throughout Australia. Chronic conditions such as cardiovascular disease, diabetes, chronic respiratory disease, and cancer are responsible for $70 \%$ of the health gap (ill health and mortality) between Aboriginal and non-Aboriginal people in terms of disability adjusted life years. ${ }^{1,2}$

The Aboriginal Health Promotion Program was developed in 1986. The aim of the Program is to prevent the onset of chronic disease and injury in the Aboriginal population in NSW. The program has primarily administered grants to Aboriginal Community Controlled Health Services and Local Health Districts to support Aboriginal health promotion projects addressing key local and state priorities.

An example of a project funded under the program was the Shake-a-leg Program, a schools-based health promotion program targeting primary and secondary schools in the Hunter New England region. The program aimed to reduce preventable health conditions in Aboriginal children through better understanding of disease processes and prevention in the home environment. An evaluation found a noticeable change in the health behaviours of the children and increased knowledge of parents about the program.

Reviews undertaken in 2007 and 2010 recommended refocusing the Aboriginal Health Promotion Program to align with the key priorities of the National Partnership Agreement on Closing the Gap in Indigenous Health Outcomes and the National Partnership Agreement on Preventive Health. The reviews identified that a range of promising and innovative projects were being implemented under the Program but that a number of issues were affecting its overall performance and success. These issues included a lack of program logic and insufficient evaluation of health promotion interventions which could identify the impact of the projects on health gains, health outcomes and workforce capacity.

Operational Guidelines and Funding Guidelines were developed to support the implementation of the Program. These guidelines outlined specific program standards and funding requirements of the Program, and provided practical guidance on the application of these standards in the planning, design, delivery and evaluation of health promotion projects.

The Centre for Aboriginal Health conducted a series of Aboriginal health promotion capacity building workshops across NSW in 2010 which focused on program logic modelling, health promotion practice and building evidence through rigorous evaluation. The majority of those consulted during these workshops considered the current program's funding allocations were too small and often used to fund one-off, ad hoc programs which contributed little to substantial and sustainable health improvements.

Based on the reviews and implementation experience at a strategic and operational level, the following policy questions require careful consideration if the Program is to achieve a more sustainable contribution to improve Aboriginal health outcomes. Firstly, based on a modelled cost benefit analysis of the Program, what would provide best gains from the expenditure of the Aboriginal Health Promotion Program fund? Secondly, what contribution would either targeted allocations or small and flexible funds to support local work make? Finally, should investment be targeted towards building the Aboriginal health 
promotion workforce including the capacity to provide interventions at the local level?

\section{References}

1. Vos T, Barker B, Stanley L, Lopez AD. The burden of disease and injury in Aboriginal and Torres Strait Islander peoples.
Brisbane: School of Population Health, The University of Queensland, 2007.

2. Zhao Y, Dempsey K. Causes of inequality in life expectancy between Indigenous and non-Indigenous people in the Northern Territory 1981-2000: a decomposition analysis. Med J Aust 2006; 184: 490-4.

\section{The Chronic Care Service Enhancement Program}

\author{
Maurice Terare $^{\mathrm{A}}$, Catriona McDonnell ${ }^{\mathrm{A}}$ \\ and Geraldine Wilson ${ }^{\mathrm{A}}$ \\ ${ }^{\mathrm{A}}$ Centre for Aboriginal Health, NSW Ministry of Health
}

The disparity in life measured in health outcomes and life expectancy between Aboriginal and non-Aboriginal people has been well documented. Aboriginal people living in NSW have a significantly higher incidence of chronic disease than the non-Aboriginal population. Chronic conditions such as cardiovascular disease, diabetes, chronic respiratory disease, and cancer are responsible for $70 \%$ of the health gap (ill health and mortality) between Aboriginal and non-Aboriginal people in terms of disability adjusted life years. ${ }^{1}$

In response to this, the NSW Ministry of Health is funding the Living Well: the NSW Aboriginal Health Chronic Care Initiative for the development, implementation and evaluation of a range of evidence-based and culturally responsive secondary prevention and chronic disease management initiatives for Aboriginal people in NSW. The target group for the Living Well Initiative is Aboriginal people aged 15 years and over diagnosed with, or at risk of developing, the chronic conditions of diabetes, cardiovascular disease, chronic respiratory disease, renal disease, musculoskeletal and connective tissue disorders and malignant cancer.

The Chronic Care Service Enhancement Program is the most significant investment of the projects funded under the Living Well Initiative. The Program aims to improve access to secondary prevention health services, as well as improving the coordination and management of care for Aboriginal people with, or at risk of, chronic diseases. The Program will enhance chronic care services being delivered under the Chronic Care for Aboriginal People Program by the Aboriginal Community Controlled Health Services (ACCHS) and Local Health Districts throughout NSW.

Nine ACCHS are receiving funding under the Program to implement strategies that will increase the accessibility of services, provide early intervention for clients at risk of chronic disease, and improve the care coordination and management of Aboriginal people with chronic diseases. The Centre for Aboriginal Health has a close working partnership with each service and the Aboriginal Health and Medical Research Council of NSW to ensure the Program is responsive to the needs of each service and local communities.

The ACCHS are implementing a range of specific strategies to improve the reach, screening and management of chronic disease in Aboriginal communities. To increase the number of Aboriginal people accessing the service for the first time or re-engaging with the service, the health services are holding open days and information sessions for local communities, providing outreach services and transport, and improving referrals with other services providers. To increase the number of clients who are screened for chronic diseases in line with evidence-based guidelines, services are providing staff training, increasing opportunistic screening, and improving reminder and recall systems. To improve the management of chronic disease in accordance with best practice guidelines, services are undertaking strategies such as organising regular multidisciplinary team case management meetings, improved care planning and referrals, delivery of self-management strategies and brief interventions to support patients.

Using the program funding, the health services have recruited a range of health professionals to implement these strategies, including Nurse Practitioners, Enrolled Nurses and Aboriginal Health Workers. Services are also able to use funding to source additional services, such as allied health and specialist services, and transport.

The second component of the Program focuses on the Local Health Districts, enhancing funding to the Chronic Care for Aboriginal People Program. A dedicated chronic care clinical position is being established in each Local Health District to provide clinical follow-up to Aboriginal patients with chronic disease within 48 hours of being discharged from hospitals across NSW.

A comprehensive evaluation of both phases of the Program is being carried out by the University of 
Newcastle to measure the effectiveness and acceptability of interventions being delivered in improving health systems as well as improving measurable health outcomes.

\section{Reference}

1. Vos T, Barker B, Stanley L, Lopez AD. The burden of disease and injury in Aboriginal and Torres Strait Islander peoples. Brisbane: School of Population Health, The University of Queensland, 2007.

\section{Responding to family violence in Aboriginal communities: The NSW Aboriginal Family} Health Strategy

\author{
Helen Gardiner ${ }^{\mathrm{A}}$ and Geraldine Wilson ${ }^{\mathrm{A}}$ \\ ${ }^{\mathrm{A}}$ Centre for Aboriginal Health, NSW Ministry of Health
}

Family violence has a devastating impact on the health and wellbeing of Aboriginal communities. ${ }^{1}$ In 2008, there were six times more Aboriginal than non-Aboriginal women reported to be victims of domestic violence (NSW Health data request on number of Indigenous victims of domestic violence related assault recorded by the NSW Police, NSW Recorded Crime Statistics from July 2006 to June 2009, from NSW Bureau of Crime Statistics and Research). The number of child protection reports made to the NSW Department of Community Services for Aboriginal children and young people has increased by more than three fold in the past 8 years. ${ }^{2}$

The Aboriginal Family Health Strategy, ${ }^{3}$ first released in 1998 presented an innovative approach to working to address family violence within a cultural context. The Strategy was originally limited to the individual and family support activities of Aboriginal Family Health Workers, including initial crisis support, advocacy and referral. Reviews of the Strategy identified a number of challenges including: the complexity of family violence; workforce shortages; community expectations; lack of consistent and coordinated service delivery; and the risk of duplicated effort due to the numerous interagency and whole of Government strategies targeting some locations.

A revised Aboriginal Family Health Strategy, released on National Sorry Day 2011, is being integrated into the NSW Health system as a model of care (Figure 1). ${ }^{4}$ The focus is family and culture with four elements: effective service delivery, strong community capacity, culturally competent work force and strategic leadership. It is built on a healing approach and is informed by research and evidence. The application of this model into mainstream services provides practical ways to enhance efforts within a culturally competent framework.

\section{Progress}

Effective service delivery: Currently 25 Aboriginal Family Health Workers are employed in prioritised areas of need, predominantly in Aboriginal Community Controlled Health Services (ACCHS) in regional NSW, and one position in the Justice Health system.

Strong community capacity: Locating Aboriginal Family Health Workers in ACCHS is in keeping with the philosophy of the strategy that 'solutions to family violence in Aboriginal communities will be community devised, managed and implemented'. These workers are now focusing more on early intervention and education which, when combined with a healing process, aims to build strong and resilient communities.

Culturally competent workforce: The Education Centre Against Violence supports the work of the Strategy to enhance the capacity of the Aboriginal workforce, and

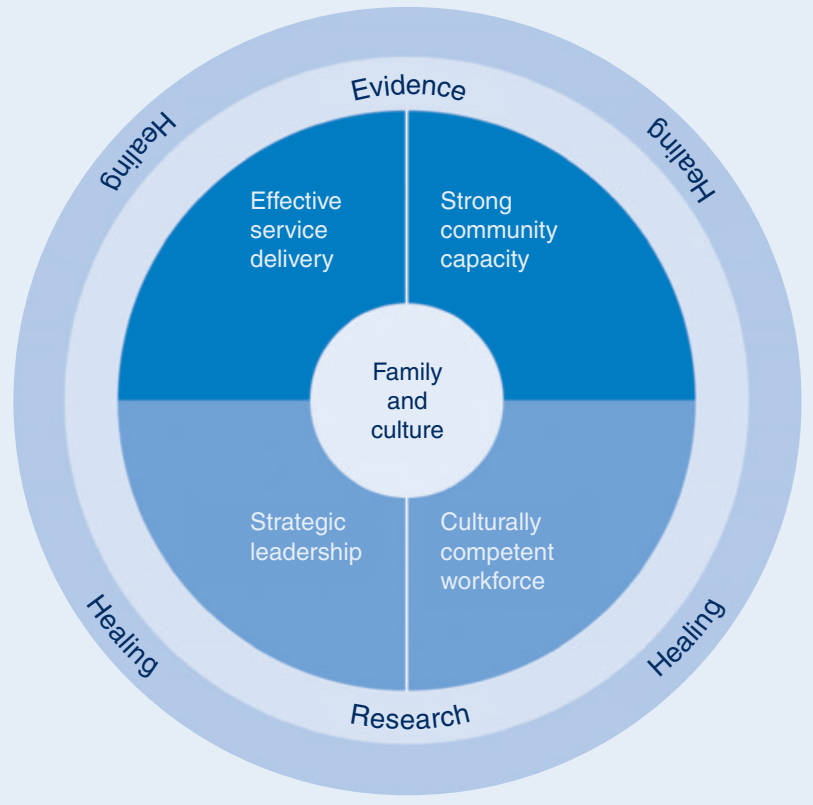

Figure 1. Aboriginal Family Health model of care Source: Aboriginal Family Health Strategy 
addresses recruitment and retention issues. The Certificate IV in Aboriginal Family Health and the NSW Advanced Diploma of Aboriginal Specialist Trauma Counselling ensure Aboriginal workers are equipped to address the complex needs of Aboriginal families. The Centre also provides training to develop the cultural competency of the non-Aboriginal workforce.

Strategic leadership: A trial of Aboriginal Family Health Coordinators, located in four Local Health Districts, is being undertaken. These coordinators will undertake a strategic role to facilitate better access for Aboriginal families to services responding to issues of family violence, including child protection.

Monitoring and evaluation: Information from the Aboriginal Family Health Worker Data Collection and preliminary evaluation of the Aboriginal Family Health Coordinator trial highlight the diverse needs of communities and the difficulty of evaluating a program based on a holistic approach that emphasises healing. Formal evaluation of the Aboriginal Family Health Strategy is planned to commence in 2012. In the long term, the success of this Strategy will be assessed from a family and community-based perspective, in accordance with the principles of Aboriginal health.

\section{References}

1. Government NSW. Discussion Paper on NSW Domestic and Family Violence Strategic Framework. NSW Department of Premier and Cabinet, Office for Women's Policy; 2009. Available at: http://www.dpc.nsw.gov.au/_data/assets/ pdf_file/0005/75533/Discussion_Paper_on_NSW_Domestic_ and_Family_Violence_Strategic_Framework.pdf (Cited 5 December 2011).

2. NSW Department of Community Services. Child Protection Quarterly Report July 2006-December 2007. Table 1.5 p 7. Available at: http://www.community.nsw.gov.au/docswr/_ assets/main/documents/quarterlyjul06_dec07.pdf (Cited 5 December 2011).

3. Health NSW. NSW Aboriginal Family Health Strategy. Sydney: NSW Department of Health; 2002.

4. Health NSW. Aboriginal Family Health Strategy 2011-2016. Responding to family violence in Aboriginal communities. Sydney: Centre for Aboriginal Health; 2011. Available at: http://www.health.nsw.gov.au/pubs/2011/aboriginal_family_ health_.html (Cited 5 December 2011).

\section{Healthy Ears, Happy Kids: a new approach to Aboriginal child ear health in NSW}

\author{
Paul Huntley ${ }^{\mathrm{A}}$, Brian Woods ${ }^{\mathrm{B}}$ and Sian Rudge $\mathrm{C}^{\mathrm{C}}$ \\ ${ }^{\mathrm{A}}$ Centre for Aboriginal Health, NSW Ministry of Health \\ ${ }^{\mathrm{B}}$ Mental Health and Drug and Alcohol Office, NSW Ministry \\ of Health \\ ${ }^{\mathrm{C}}$ Sax Institute
}

Otitis media is one of the most prevalent childhood conditions in developing countries and contributes to excess childhood mortality. ${ }^{1}$ For children in developed countries, otitis media usually resolves spontaneously and without antibiotic treatment. In developing countries or in communities subject to socio-economic disadvantage, including many Australian Aboriginal communities, acute instances of the condition often do not resolve and may recur repeatedly. ${ }^{2}$ Children with conductive hearing loss associated with otitis media may experience serious interruption to speech and language development and disrupted learning in school. For other children this can further exacerbate other educational and social disadvantages. ${ }^{1}$

To address ear health issues for Aboriginal children in NSW under the National Partnership Agreement for Closing the Gap in Indigenous Health Outcomes initiative, NSW Health has developed and released the NSW Aboriginal Ear Health Program Guidelines 2011-2015.
The NSW Otitis Media Screening Program for 0-6 year old Aboriginal children was the NSW Health approach to ear health in Aboriginal children from 2004 to 2008. This program was a population-based (near universal) screening initiative to identify and treat young Aboriginal children with otitis media. The Centre for Aboriginal Health commissioned an evaluation of the effectiveness and appropriateness of this program in 2008. The evaluation involved extensive consultation across the sector through case studies and interviews with frontline health workers and policy makers from Aboriginal Community Controlled Health Services, the Department of Education and Training, the former Department of Health, the Office for Aboriginal and Torres Strait Islander Health, and the former Area Health Services.

The evaluation found that the program did not address the underlying social and environmental factors contributing to ear disease, was unsupported by evidence, was cost prohibitive and did not reduce prevalence. ${ }^{3}$ It recommended discontinuing the existing approach and adopting a broad public health approach that integrated the ear health program with existing child health surveillance and health-care programs, such as Child Health Checks, the Personal Health Record (Blue Book), the Aboriginal 


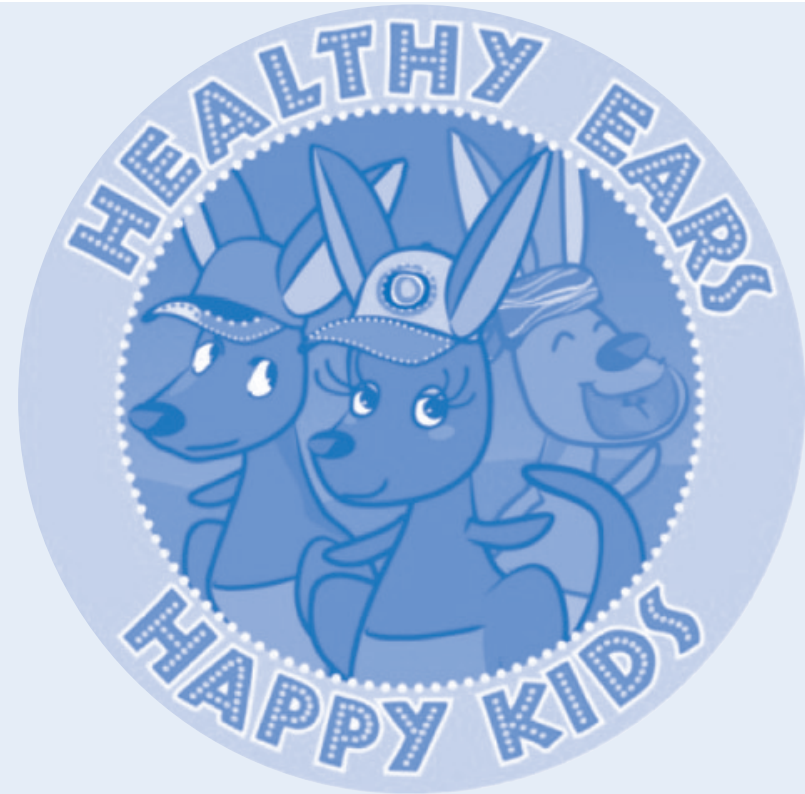

Figure 1 The Healthy Ears Happy Kids logo

and Maternal Infant Health Strategy, the Aboriginal Oral Health Program, Statewide Eyesight Pre-schooler Screening, Statewide Infant Screening Hearing (SWISH) and Building Stronger Foundations for Aboriginal Children. The findings and recommendations of the evaluation are in keeping with a recent evidence review which stated that ... the treatment of children detected through repeated screening has been shown to provide no long-term developmental or academic benefit. ${ }^{4}$

To implement the recommendations of the evaluation, NSW Health established the NSW Otitis Media Expert Advisory Committee, comprised of a multidisciplinary team of health and education professionals with relevant ear health expertise. In 2011 the Advisory Committee and the Centre for Aboriginal Health released the NSW Aboriginal Ear Health Program Guidelines 2011-2015. ${ }^{5}$ The aim of these guidelines is to reduce the number of young Aboriginal children aged under 5 years being affected by otitis media, by delivering a strong preventive approach through better education for parents, carers, extended families, health and education professionals.

The key actions of the program aim to reduce prevalence of otitis media by:

- addressing environmental health risk factors

- reducing maternal ante-natal smoking

- increasing maternal post-natal breastfeeding;

- improving safe and healthy housing conditions

- linking with existing child health surveillance programs;

- improving awareness and education among the Aboriginal community and human services professionals.

Ear health promotion resources have also been developed to support the new approach using the campaign branding Healthy Ears Happy Kids (Figure 1). With its new broad public health approach and strong emphasis on prevention, the program is committed to improving the ear health of young Aboriginal children across the state.

\section{References}

1. Couzos S, Metcalf S, Murray R. Systematic review of existing evidence and primary care guidelines on the management of otitis media in Aboriginal and Torres Strait Islander populations. Canberra: Commonwealth Department of Health and Family Services. Office for Aboriginal and Torres Strait Islander Health Services; 2001. Available at: http://www.health.gov.au/oatsih/ pubs/omp.htm (Cited 6 March 2012).

2. Gibney KB, Morris PS, Carapetis JR, Skull SA, Smith-Vaughan $\mathrm{HC}$, Stubbs E et al. The clinical course of otitis media in high-risk Australian Aboriginal children: a longitudinal study. $B M C$ Pediatr 2005; 5: 16. doi:10.1186/1471-2431-5-16

3. ARTD Pty Ltd. Evaluation of the Aboriginal Otitis Media Screening Program Final Report to the NSW Department of Health. 2008. Available at: http://www.health.nsw.gov.au/pubs/ 2008/otitis_report.html (Cited 6 March 2012).

4. Morris P, Hopkins S. Rapid Evidence Summary - Does ear health screening improve outcomes in young children? 2009. Available at: http://www.healthinfonet.ecu.edu.au/other-health-conditions/ ear/reviews/other-reviews (Cited 6 March 2012).

5. NSW Ministry of Health. NSW Aboriginal Ear Health Program Guidelines 2011-2015. 2011. Available at: http://www.health. nsw.gov.au/policies/gl/2011/GL2011_013.html (Cited 6 March 2012).

\section{Reporting of Aboriginal collections in NSW}

\author{
Louise Maher ${ }^{\mathrm{A}}$, Caroline Turnour ${ }^{\mathrm{A}}$ \\ and Jessica Stewart ${ }^{\mathrm{B}}$ \\ ${ }^{\mathrm{A}}$ Centre for Aboriginal Health, NSW Ministry of Health \\ ${ }^{\mathrm{B}}$ Centre for Epidemiology and Evidence, NSW Ministry of Health
}

The accurate reporting of Aboriginal people in administrative data sets is essential to effectively monitor progress towards closing the gap in Aboriginal health outcomes. Improving the reporting of Aboriginal people in NSW Health's data collection systems is a key priority under the National Partnership Agreement on Closing the Gap in Indigenous Health Outcomes. ${ }^{1}$

The National best practice guidelines for collecting Indigenous status in health data sets documents the 
recommended national approach for collecting and recording accurate information on the Aboriginal status of clients. ${ }^{2}$ Self-report in response to a standard question is described as the most accurate means of ascertaining whether a person is Aboriginal or not. The question is: Are you (is the person) of Aboriginal or Torres Strait Islander origin?

In NSW, there is under-reporting of Aboriginal people across a range of health data collections and systems. In the NSW Admitted Patient Data Collection it is estimated that the level of correct reporting is $90.7 \%{ }^{3}$ The under-reporting of Aboriginal people in NSW Health's data collection systems may be attributed to a range of factors, including a lack of staff awareness and training, staff reluctance to ask the question, client reluctance to answer the question, staff perceptions about whether people want to identify as Aboriginal, lack of privacy when answering the question, limited data validation and follow-up of missing information, and inadequate data management systems. ${ }^{4}$ Aboriginal people's willingness to disclose their Aboriginal status and administrative staff preparedness to ask the question also depends on the existence of a culturally safe environment.

The Australian Council on Healthcare Standard Evaluation and Quality Improvement Program (EQuIP 5) requires all hospitals to demonstrate action on standards aimed at improving Aboriginal identification. This requirement provides an opportunity to support system-wide improvement in Aboriginal health and hospital data. A national pilot project in five hospitals has demonstrated that using a continuous quality improvement framework to improve the culture of hospitals for Aboriginal people can result in improving identification of Aboriginal people in health data sets. ${ }^{5}$

NSW Health currently has a number of activities underway to improve the accuracy of reporting of Aboriginal people in administrative datasets:

- The Centre for Aboriginal Health is reviewing the policy on identification of Aboriginal people to ensure it is consistent with the National Best Practice Guidelines and supports the EQuIP5 accreditation framework, and will support Local Health Districts in the implementation and monitoring of the revised policy. ${ }^{6} \mathrm{NSW}$ Health is also working collaboratively with the Australian Institute of Health and Welfare to conduct the 2011 Admitted Patient Indigenous Status Survey in NSW.

- The Centre for Epidemiology and Evidence is investigating the feasibility of using record linkage to estimate the under-reporting of Aboriginal people on routinely collected NSW Health datasets. The project uses linked records from a number of data collections, and has developed algorithms to enhance Aboriginal identification within each data collection.

- The NSW Hospital Identification Project, currently being developed by the Centre for Aboriginal Health, aims to demonstrate the effectiveness of a continuous quality improvement framework in improving the cultural competency of eight hospitals. The project aims to address the under-reporting of Aboriginal people by adopting a system-wide approach that goes beyond staff training and improvements in administrative systems to include initiatives that promote the cultural competency of each hospital.

Improved identification will lead to more accurate reporting of disease and risk factors among Aboriginal people and stronger evaluation on health system performance in closing the gap, which in turn will support the delivery of more effective, sustainable and culturally appropriate health services for Aboriginal people in NSW.

\section{References}

1. Council of Australian Governments. National Partnership Agreement on Closing the Gap in Indigenous Health Outcomes. Canberra: COAG; 2009.

2. Australian Institute of Health and Welfare. National best practice guidelines for collecting Indigenous status in health data sets. Cat. No. AIHW 29. Canberra: AIHW; 2010.

3. Bentley JP, Taylor LK, Brandt PG. Reporting of Aboriginal and Torres Strait Islander peoples on the NSW Admitted Patient Data Collection: the 2010 Data Quality Survey. NSW Public Health Bull 2012; 23(1): 17-20.

4. Lovett R. ACT public hospital staff attitudes concerning Indigenous origin information and estimating Indigenous under-identification in ACT public hospital admission data. Chapter 4. Master of Applied Epidemiology (Indigenous Health) thesis. National Centre for Epidemiology and Public Health, Australian National University; 2006.

5. Willis J, Wilson G, Renhard R, Chong A, Clarke A. Improving the culture of hospitals project: final report. Melbourne: Australian Institute of Primary Care; 2010. Available at: http://www.lowitja.org.au/files/crcah_docs/ ICHP_Final_Report_August_2010.pdf (Cited 6 March 2012).

6. Health NSW. Aboriginal and Torres Strait Islander Origin Recording of Information of Patients and Clients. Policy Directive PD2005_547. 2005. Available at: http://www.health. nsw.gov.au/policies/pd/2005/PD2005_547.html (Cited 6 March 2012). 